\title{
UNA PIETRA MILIARE NELLA COMPRENSIONE DEL MICROCOSMO: IL BOSONE DI HIGGS
}

\author{
ORESTE NICROSINI (*) \\ Nota presentata dal m.e. Giacomo M. D'Ariano \\ (Adunanza del 7 marzo 2013)
}

Sunto. - Il Premio Nobel per la Fisica 2013 è stato conferito a P.W. Higgs e F. Englert per l'ideazione di un quadro teorico in grado di contribuire alla comprensione dell'origine della massa delle particelle subatomiche. Il meccanismo individuato (noto come meccanismo di Higgs) è stato recentemente confermato dall'osservazione della particella fondamentale da questo previsto (il cosiddetto bosone di Higgs) ad opera degli esperimenti ATLAS e CMS presso il Large Hadron Collider del CERN di Ginevra. Al risultato hanno contribuito i fisici delle particelle di tutto il mondo, teorici e sperimentali, negli ultimi trent'anni almeno: nel presente scritto si ripercorre la storia di questa scoperta, una pietra miliare della comprensione attuale del microcosmo.

$$
* * *
$$

Abstract. - The Nobel Prize in Physics 2013 has been awarded to P.W. Higgs and F. Englert for the conception of a theoretical framework that contributes to the understanding of the origin of mass of subatomic particles. The mechanism (known as the Higgs mechanism) has been recently confirmed by the observation of the fundamental particle predicted (the so called Higgs boson) by the ATLAS and CMS experiments of the Large Hadron Collider at CERN, Geneva. Particle physicists all over the world, both theoreticians and experimentalists, have contributed to the result in the last thirty years, to say the least: in the present paper an account is given of this discovery, a milestone in the current understanding of the microcosm.

(*) Istituto Nazionale di Fisica Nucleare, Sezione di Pavia, Dipartimento di Fisica, Università degli Studi di Pavia, Pavia, Italy.

E-mail: oreste.nicrosini@pv.infn.it 


\section{INTRODUZIONE}

Nell'ottobre del 2013 il fisico britannico Peter W. Higgs, attualmente professore emerito presso l'Università di Edinburgo, e il fisico belga François Englert, attualmente professore emerito alla Université Libre de Bruxelles, sono stati insigniti del Premio Nobel per la Fisica [1] con la seguente motivazione: «per la scoperta teorica di un meccanismo che contribuisce alla nostra comprensione dell'origine della massa delle particelle subatomiche, e che di recente è stato confermato attraverso la scoperta della particella fondamentale prevista, dagli esperimenti ATLAS e CMS al Large Hadron Collider del CERN».

Higgs e Englert sono entrambi fisici teorici. Gli studi fondamentali per i quali sono stati insigniti del Premio Nobel $[2,3]$ sono pressoché contemporanei, del $1964 .{ }^{1}$ D'altra parte dalla motivazione del Premio appare chiaro che la conferma sperimentale della previsione teorica, annunciata per la prima volta nel luglio del 2012 dal CERN di Ginevra, è stata condizione necessaria per l'assegnazione del Premio stesso. E la conferma sperimentale dell'esistenza del cosiddetto bosone di Higgs è il coronamento dell'attività che ha intensamente impegnato i fisici delle particelle di tutto il mondo, teorici e sperimentali, negli ultimi trent'anni almeno. Nel presente scritto si ripercorre la storia delle ricerche che hanno condotto alla scoperta della particella di Higgs, pietra miliare della comprensione attuale del microcosmo.

Fin dagli albori del pensiero razionale l'uomo si è domandato se la multiformità del reale sia riconducibile all'esistenza di "pochi" costituenti elementari della materia che ci circonda. Dai primi anni del XX secolo, dopo la scoperta degli atomi, la fisica ha iniziato a indagare il mondo subatomico e subnucleare, dapprima studiando i raggi cosmici e poi utilizzando gli acceleratori di particelle. Questi possono essere considerati potentissimi microscopi, in grado di "vedere" il mondo subnucleare e quindi fornirci informazioni sulla sua struttura. Un secolo di ricerche ci ha portato oggi a formulare quello che viene chiamato Modello Standard delle interazioni fondamentali, la teoria che descrive i costituenti "ultimi" della

1 È opportuno ricordare che il lavoro di F. Englert è in collaborazione con R. Brout, fisico teorico belga di origine statunitense, scomparso nel 2011. Inoltre intorno alla metà degli anni ' 60 anche altri studiosi stavano lavorando allo stesso problema, come documentato in [4] e [5]. 
materia e le loro interazioni [6]. Il percorso è stato spesso tortuoso, ma la teoria standard nella sua formulazione attuale è convincente e ha già passato il vaglio di numerose prove sperimentali. Così si esprime Shaldon Lee Glashow, uno dei padri del Modello Standard, nella sua Nobel Lecture del 1979: «[...] the study of elementary particles was like a patchwork quilt. [...] The theory we now have is an integral work of art: the patchwork quilt has become a tapestry». Ciò che mancava ancora, fino al luglio 2012, per accreditare il Modello Standard come completamente verificato nei suoi aspetti fondamentali era la prova sperimentale dell'esistenza del bosone di Higgs, una delle sue previsioni cruciali.

\section{I COSTITUENTI FONDAMENTALI}

Verso la fine del XIX secolo gli elementi chimici (atomi) risultano organizzati in uno schema ordinato noto come tavola periodica degli elementi, o tavola di Mendeleev dal nome del suo ideatore. In questa tavola gli elementi sono disposti secondo gruppi che presentano proprietà chimiche molto simili, pur avendo gli elementi ad essi appartenenti masse diverse. La periodicità della tavola, cioè il fatto che percorrendo una sua riga si incontrino elementi dalle proprietà chimiche via via diverse ma che si ripresentano passando alla riga successiva, è un'indicazione del fatto che gli atomi, considerati fino a quel tempo indivisibili, in realtà, a dispetto del loro etimo (dal greco ótouos, indivisibile), siano a loro volta composti di oggetti più elementari. E in effetti una serie di esperimenti condotti tra la fine dell' 800 e i primi decenni del '900 ha rivelato che all'interno degli atomi si trovano gli elettroni (J.J. Thompson, 1897) e che gli atomi sono strutture sostanzialmente vuote, la cui massa è concentrata in un nucleo atomico attorno al quale "orbitano" appunto gli elettroni (E. Rutherford, 1911). Non solo, anche il nucleo atomico a sua volta è composto di oggetti più elementari, oggi noti come protoni e neutroni (J. Chadwick, 1932). E utile richiamare le dimensioni di atomi e nuclei atomici: le dimensioni di un atomo si collocano tra 1 e 10 decimi di miliardesimi di metro; le dimensioni del nucleo atomico sono dell'ordine di un milionesimo di miliardesimo di metro. Se il nucleo dell'idrogeno (protone) fosse un pallone del diametro di un metro, l'elettrone potrebbe essere una piccola bilia che "orbita" ad una distanza di 100 chilometri circa.

È necessario attendere la fine degli anni '60 del secolo scorso per 
avere evidenza sperimentale circa il fatto che anche protone e neutrone, i costituenti del nucleo atomico, sono a loro volta oggetti composti. In un famoso esperimento realizzato al laboratorio SLAC, Stati Uniti, scagliando elettroni di alta energia su un bersaglio di protoni (idrogeno) è stata effettuata quella che può essere definita come la radiografia del protone, in una versione a più alta energia dello storico esperimento di Rutherford. Da tale esperimento risulta che protone e neutrone non sono oggetti elementari, ma sono costituiti a loro volta da altre particelle, denominate quark. Queste possiedono proprietà inusuali, come ad esempio carica elettrica frazionaria, ma le loro proprietà più appariscenti sono le seguenti: i quark sono sostanzialmente liberi di muoversi all'interno del protone/neutrone che li contiene (proprietà nota come libertà asintotica) ma non possono essere "strappati" da questo (proprietà nota come confinamento).

Come già accennato, l'indagine del mondo subnucleare è oggi condotta principalmente utilizzando acceleratori di particelle. Le ragioni di questa scelta sono sostanzialmente due. Anzitutto va detto che, in accordo con la Meccanica Quantistica, ad ogni particella materiale è associata un'onda, la cui lunghezza è tanto minore quanto maggiore è l'energia della particella; quindi particelle molto energetiche sono sensibili a dettagli di piccole dimensioni. In secondo luogo, secondo la celeberrima formula di Einstein $E=m c^{2}$ (dove $E$ è l'energia, $m$ la massa e $c$ la velocità della luce) l'energia può essere convertita in massa e viceversa. Dunque particelle molto energetiche, urtandosi e liberando la loro energia, possono creare particelle molto pesanti.

\section{LE FORZE FONDAMENTALI}

Cosa lega i costituenti fondamentali a formare la materia che conosciamo? A oggi sono note quattro forze fondamentali, mediante le quali cioè interagiscono i costituenti fondamentali.

La prima di queste forze è la gravità, cioè la forza responsabile del fatto che due masse si attirano reciprocamente. Il primo a studiarla è stato I. Newton (1643-1727) formulando la sua teoria della gravitazione universale, in grado di spiegare sia il peso dei corpi che le orbite dei pianeti. Oggi la gravitazione è descritta dalla Teoria della Relatività Generale di A. Einstein (1879-1955), secondo cui la gravità è una deformazione dello spazio-tempo generata dalle masse. 
La seconda forza fondamentale è la forza elettromagnetica, responsabile dell'interazione tra particelle dotate di carica elettrica. L'elettromagnetismo è stato studiato da molti autori (Coulomb, Volta, Ampère, Faraday per citarne solo alcuni) e sintetizzato da J.C. Maxwell (1831-1879) nelle sue famose equazioni che unificano i fenomeni elettrici e magnetici. Esso è responsabile di gran parte dei fenomeni della vita di tutti i giorni, dal fatto che possiamo vedere le persone e gli oggetti che ci circondano grazie alla luce (una forma di radiazione elettromagnetica) all'esistenza del campo magnetico terrestre, dallo scoccare dei fulmini durante un temporale al funzionamento di tutti gli apparati di cui ci serviamo quotidianamente. La teoria elettromagnetica, opportunamente combinata con la Teoria della Relatività Ristretta di Einstein e con la Meccanica Quantistica, è oggi nota come Elettrodinamica Quantistica, sviluppata da vari autori attorno agli anni ' 50 del Novecento, ed è la teoria di gran lunga più precisa che possediamo. Due tra le sue previsioni più importanti, puntualmente verificate dagli esperimenti, sono le seguenti: le particelle cariche interagiscono scambiandosi "granuli" di energia elettromagnetica, chiamati fotoni o quanti di luce, e oltre alle particelle di materia esistono anche le antiparticelle, in tutto e per tutto identiche alle particelle tranne che per la carica. Così, oltre all'elettrone esiste anche il positrone, l'antiparticella dell'elettrone, e così via.

Sia la forza di gravità che quella elettromagnetica sono forze a lungo raggio d'azione. Questa è la ragione per cui sono state scoperte e studiate per prime, manifestandosi a livello macroscopico. Esistono però anche altre due forze fondamentali, a brevissimo raggio d'azione (dell'ordine delle dimensioni del nucleo atomico).

La prima di queste è la forza nucleare forte, responsabile del fatto che protoni e neutroni si possono aggregare a formare i nuclei atomici ovvero, a livello più elementare, i quark si aggregano a formare protoni e neutroni. La descrizione contemporanea della forza nucleare forte è fornita dalla Cromodinamica Quantistica, una teoria costruita all'inizio degli anni '70 del secolo scorso generalizzando opportunamente l'Elettrodinamica Quantistica, la quale prevede che i quark siano legati all'interno di protoni e neutroni da particelle analoghe ai fotoni chiamate gluoni (da glue, colla).

La seconda di queste è la forza nucleare debole, responsabile ad esempio di gran parte dei fenomeni radioattivi e della fusione nucleare che avviene all'interno delle stelle, il nostro sole compreso. Una tipica reazione di interazione nucleare debole è ad esempio quella in cui un 
protone $(\mathrm{p})$ si trasforma in un neutrone $(\mathrm{n})$ rilasciando un positrone $\left(\mathrm{e}^{+}\right)$ e un neutrino (n). Successivamente alla trasformazione, il neutrone può legarsi, mediante la forza nucleare forte, ad un altro protone per formare il nucleo di deuterio.

Oggi la forza nucleare debole è unificata a quella elettromagnetica nella Teoria dell'Unificazione Elettrodebole, proposta da S.L.Glashow, A. Salam e S. Weinberg alla fine degli anni '60 del secolo scorso e sviluppata successivamente da molti autori. Secondo questa teoria, come la forza elettromagnetica è trasmessa dallo scambio di fotoni, così la forza debole è trasmessa dallo scambio di nuove particelle, il $W$ e lo $Z$, scoperte dalle Collaborazioni UA1 e UA2 al CERN di Ginevra nel 1983. Per l'ideazione e la realizzazione dell'acceleratore che ha consentito la scoperta, Carlo Rubbia e Simon van der Meer sono stati insigniti del Premio Nobel per la fisica 1984.

\section{Il CAMPO Di Higgs E IL BOSONE Di HigGS}

La Cromodinamica Quantistica e la Teoria dell'Unificazione Elettrodebole costituiscono, nel loro insieme, quello che oggi viene definito Modello Standard delle Interazioni Fondamentali. Questo è stato sottoposto, nel corso degli anni, a numerosissime prove sperimentali, soprattutto al CERN di Ginevra dove tra la fine degli anni ' 80 e per tutti gli anni '90 del secolo scorso è stato in funzione l'acceleratore LEP (Large Electron Positron collider) e al Fermilab di Chicago dove, dalla fine degli anni '80 del secolo scorso a circa il 2010, ha operato l'acceleratore TEVATRON. In particolare l'acceleratore LEP, facendo scontrare elettroni e positroni ad altissima energia (dell'ordine di 100 miliardi di elettronvolt, cioè $100 \mathrm{GeV}$, corrispondenti a circa 100 volte la massa di un protone, ma concentrata in una regione di raggio dell'ordine di un miliardesimo di miliardesimo di metro), ha prodotto un gran numero di particelle $Z$ e $W$, consentendo approfondite analisi sperimentali sul Modello Standard, tutte superate con successo.

Una previsione cruciale del Modello Standard ha trovato conferma definitiva solo recentemente: l'esistenza del bosone di Higgs, una particella molto elusiva ma fondamentale dal punto di vista della consistenza matematica del Modello Standard, ipotizzata da F. Englert, P. Higgs come risultato di un fenomeno noto tra gli addetti ai lavori come rottura spontanea della simmetria. 
Qual è dunque il ruolo del bosone di Higgs all'interno del Modello Standard? Va detto che questa teoria, se la si limita solo alle particelle già citate come elettrone, quark, $W$ e $Z$, è calcolabile a condizione che nessuna delle particelle possegga massa, cioè che le particelle siano costrette, secondo la Relatività Ristretta, a muoversi perennemente alla velocità della luce. Ma noi sappiamo che le particelle in questione (tranne i fotoni e i gluoni) posseggono massa. Ebbene il ruolo del campo di Higgs è proprio quello di generare la massa delle particelle elementari che con esso interagiscono, preservando la coerenza matematica, e la calcolabilità, della teoria.

Come accade questo? L'universo, secondo Higgs, è permeato ovunque dal campo di Higgs. Le particelle, muovendosi in questa sorta di "fluido", sono come "frenate", si muovono a velocità inferiore alla velocità della luce e dunque, ancora per la teoria della Relatività Ristretta, acquistano massa. Per alcune di queste particelle, come i fotoni, il fluido è completamente trasparente: queste non avvertono alcun effetto, continuano a muoversi alla velocità della luce e dunque non acquistano massa. Il meccanismo appena delineato è illustrato in Fig. 1.

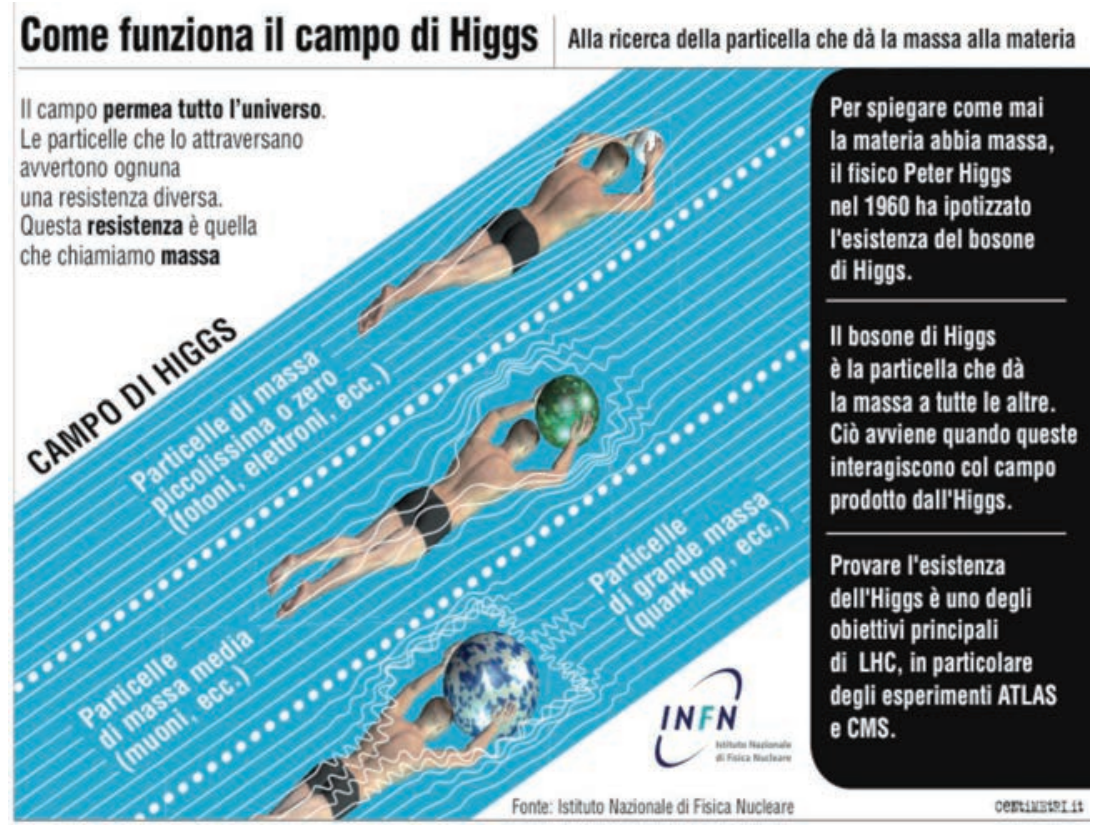

Fig. 1 - Illustrazione del funzionamento del meccanismo di Higgs (fonte INFN). 
E cos'è il bosone di Higgs? Altro non è che un'onda in questo fluido che, in accordo con la Meccanica quantistica, a noi si manifesta come particella. Il Modello Standard è in grado di prevedere tutte le proprietà di questa particella, tranne la sua massa che è uno dei parametri liberi della teoria.

\section{LA NOSTRA TAVOLA PERIODICA}

Possiamo a questo punto introdurre la "tavola periodica" come risulta dalle ricerche condotte in particolare a partire dalla fine degli anni '60 del secolo scorso. Essa è mostrata in Tab. 1. La prima parte della Tabella mostra le particelle "materia" mentre la seconda elenca le particelle responsabili della trasmissione delle forze fondamentali. La materia che ci circonda è costituita dalle particelle della prima colonna: $i$ quark $u$ e $d$ sono i costituenti di protoni e neutroni, gli elettroni fanno parte della struttura di atomi e molecole, la particella $v_{\mathrm{e}}$ è il neutrino elettronico che accompagna i decadimenti radioattivi causati dalla forza nucleare debole. Le particelle $\mu$ e $v_{\mu}$ della seconda colonna costituiscono la gran parte dei raggi cosmici che arrivano a terra. Tutte le altre particelle sono state prodotte in laboratorio. Per quanto riguarda le particelle "forza", $\gamma$ è il fotone responsabile della forza elettromagnetica, $g$ rappresenta i gluoni responsabili della forza nucleare forte, $W$ e $Z$ mediano la forza nucleare debole. La particella chiamata gravitone è ipotizzata mediare l'interazione gravitazionale; per il momento non esistono evidenze sperimentali che ne suggeriscano l'esistenza. E infine è riportata anche la particella di Higgs, con un punto interrogativo per il fatto che le risultanze sperimentali odierne, pur rappresentando una convincente evidenza della sua esisten$\mathrm{za}$, vanno consolidate da approfondite analisi quali quelle che si svolgeranno nei prossimi anni. Tutte le particelle osservate risultano "puntiformi", prive di struttura, fino a $10^{-19}$ metri, un decimo di miliardesimo di miliardesimo di metro, la minima distanza oggi esplorabile mediante gli acceleratori di particelle.

Il Modello Standard delle interazioni fondamentali appena delineato, pur sottoposto a stringenti prove sperimentali tutte superate con esito positivo, non può essere la Teoria finale che descrive la materia a tutte le scale di lunghezza. Infatti esso non include nella sua formulazione la forza di gravità, che pur ovviamente esiste, e non è in grado di render conto di risultati sperimentali/osservativi recentemente acquisi- 
ti. Il primo di questi riguarda il fenomeno noto come oscillazione di neutrini. Per un effetto dovuto alla Meccanica Quantistica, infatti, i neutrini possono trasformarsi gli uni negli altri: così ad esempio, i neutrini elettronici possono divenire neutrini del $m u$, e a loro volta i neutrini del $m u$ possono trasformarsi in neutrini del tau (fenomeno recentemente osservato dall'esperimento OPERA, collaborazione tra il CERN e i Laboratori del Gran Sasso dell'Istituto Nazionale di Fisica Nucleare). Affinché questo avvenga i neutrini devono essere dotati di massa (seppure molto piccola), il che richiede di prendere in considerazione opportune estensioni del Modello Standard. Inoltre da varie osservazioni astronomiche, iniziate già negli anni ' 30 del secolo scorso, risultano due fatti piuttosto sorprendenti: per spiegare le caratteristiche di rotazione delle galassie è necessario ipotizzare l'esistenza di una nuova forma di materia, a noi sconosciuta, nota con il nome di materia oscura; inoltre l'espansione dell'Universo appare accelerata, e questo fatto può essere spiegato introducendo una nuova forma di energia, anch'essa a noi sconosciuta, che va sotto il nome di energia oscura. Per quanto riguarda la materia oscura, un'opportuna estensione del Modello Standard, nota come Supersimmetria, fornisce un candidato naturale, una specie di neutrino molto pesante chiamato neutralino. Per quanto riguarda viceversa l'energia oscura, al momento non esistono spiegazioni plausibili, e accreditate unanimemente dalla comunità scientifica, all'interno delle teorie che conosciamo.

Tab. 1 - La nostra "tavola periodica".

\begin{tabular}{llllllll}
\hline \multicolumn{2}{l}{ Famiglie di particelle } & & & & \\
& \multicolumn{2}{l}{ Prima famiglia } & \multicolumn{2}{l}{ Seconda famiglia } & \multicolumn{2}{l}{ Terza famiglia } \\
\hline Quark & $u$ & quark up & $c$ & quark charm & $t$ & quark top \\
& $d$ & quark down & $s$ & quark strange & $b$ & quark bottom \\
\hline \multirow{2}{*}{ Leptoni } & $v_{\mathrm{e}}$ & neutrino el. & \multicolumn{2}{c}{$v_{\mu}$} & neutrino mu. & $v_{\tau}$ & neutrino tau \\
& $e$ & elettrone & $\mu$ & muone & \multicolumn{2}{c}{$\tau$} & tau \\
\hline
\end{tabular}

Mediatori di forza

$\gamma$ fotone Forza elettromagnetica (atomi, chimica, luce...)

g gluone Forza nucleare forte (struttura protone e neutrone)

$Z$ e W bosoni deboli Forza nucleare debole (decadimento beta, combustione stelle)

G (?) gravitone Forza gravitazionale (ipotetico)

H (?) bosone di Higgs Massa delle particelle (recentemente osservato, proprietà sotto indagine dettagliata) 


\section{LA RICERCA SPERIMENTALE E LA SCOPERTA}

"Il gran libro della Natura è scritto in caratteri matematici", ci ha insegnato Galileo Galilei. E per poterlo capire è necessario mettere a confronto le "sensate esperienze" da una parte, cioè gli esperimenti in laboratorio, e le "certe dimostrazioni" dall'altra, cioè le previsioni che si possono ricavare da una teoria. Solo dal confronto tra le previsioni di una teoria e il relativo riscontro sperimentale è possibile stabilire se la teoria descriva correttamente la realtà, oppure vada semplicemente scartata. Il principale laboratorio dove il Modello Standard è stato ed è sottoposto a verifica sperimentale è il CERN di Ginevra. Qui tra la fine degli anni '80 del secolo scorso e l'anno 2000 ha operato l'acceleratore LEP, mediante il quale fasci di elettroni e positroni (le antiparticelle degli elettroni) erano portati a collidere con un'energia tale da produrre copiosamente i bosoni dell'interazione elettrodebole, $W$ e $Z$. Macchina di precisione straordinaria, il LEP ha consentito di studiare i bosoni elettrodeboli in estremo dettaglio, tanto che è stato possibile mettere in evidenza effetti quantistici previsti dalla teoria [7]. Questi hanno consentito di prevedere con buona accuratezza la massa allora ignota del quark top (scoperto nel 1995 all'acceleratore TEVATRON, acceleratore protone-antiprotone presso il Fermilab di Chicago, di massa compatibile a quella indicata dal LEP) e la "finestra di massa" possibile per il bosone di Higgs. Tale finestra fornisce un valore stimato per la massa del bosone di Higgs compreso all'incirca tra 115 e $160 \mathrm{GeV}$.

Nello stesso tunnel del LEP, di circa ventisette chilometri di circonferenza che corre nel sottosuolo della campagna svizzera e francese a una profondità di circa un centinaio di metri, è recentemente entrato in funzione l'LHC (il Large Hadron Collider), che accelera e porta a collidere protoni contro protoni ad un'energia mai raggiunta prima in laboratorio (a regime si tratterà di $14 \mathrm{TeV}$, migliaia di miliardi di elettronvolt).

Uno degli obiettivi dell'LHC è proprio chiarire se il bosone di Higgs esista e quali siano le sue proprietà. In un collisore adronico, quale l'LHC, il bosone di Higgs può essere prodotto in diversi modi (fusione di gluoni o bosoni vettoriali, produzione associata con bosoni vettoriali o coppie di quark top-antitop). Una volta prodotto, il bosone di Higgs, particella estremamente instabile, decade in vari possibili modi di decadimento: in coppie di quark $b$; in coppie di gluoni; in coppie di bosoni vettoriali deboli che a loro volta decadono in quattro par- 
ticelle di materia, per esempio una coppia elettrone-positrone e una coppia muone-antimuone; in coppie di fotoni.

Tra tutti questi processi, quello principalmente utilizzato per la ricerca è decritto in Fig. 2. La collisione tra protoni, oggetti come sappiamo composti di quark e gluoni, può produrre la "fusione" di due gluoni in una particella di Higgs, che a sua volta può decadere in coppie di fotoni. Dall'analisi delle energie e degli angoli a cui vengono emessi i due fotoni è possibile risalire alla massa e ad altre proprietà della particella di Higgs. Un tipico evento di questo genere come registrato dai rivelatori è mostrato in Fig. 3.

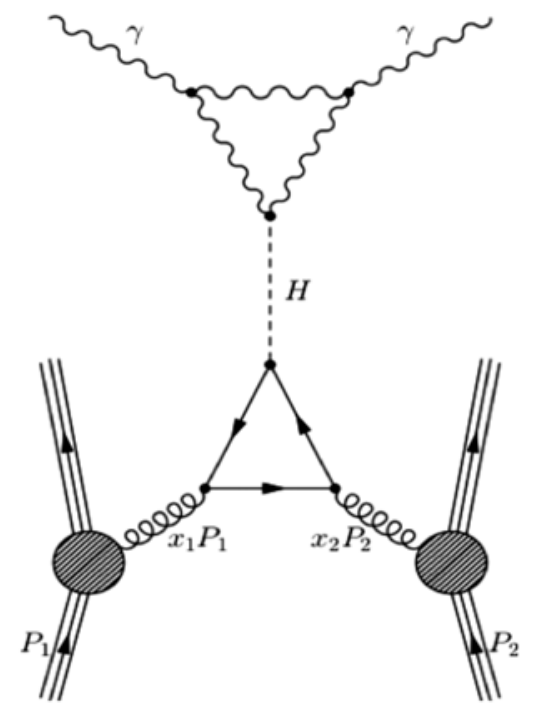

Fig. 2 - Il processo principale per la ricerca del bosone di Higgs.

Il 4 luglio del 2012, durante un seminario diramato in mondovisione dal CERN presenti F. Englert e P. Higgs tra il pubblico, i rappresentanti dei due principali esperimenti (ATLAS e CMS, collaborazioni costituite da migliaia di fisici provenienti da tutto il mondo) hanno comunicato di avere evidenza sperimentale circa l'esistenza di una particella che, a quanto risulta dalle prime analisi, possiede caratteristiche compatibili con quelle previste per il bosone di Higgs, con una massa corrispondente a circa $125 \mathrm{GeV}[8,9]$. 
È solo l'inizio di un lungo percorso che deve chiarire se effettivamente tutte le proprietà della particella osservata coincidano con quelle attese per il bosone di Higgs oppure no, e che fornirà informazioni sulla fisica che sappiamo deve esistere "oltre il Modello Standard".

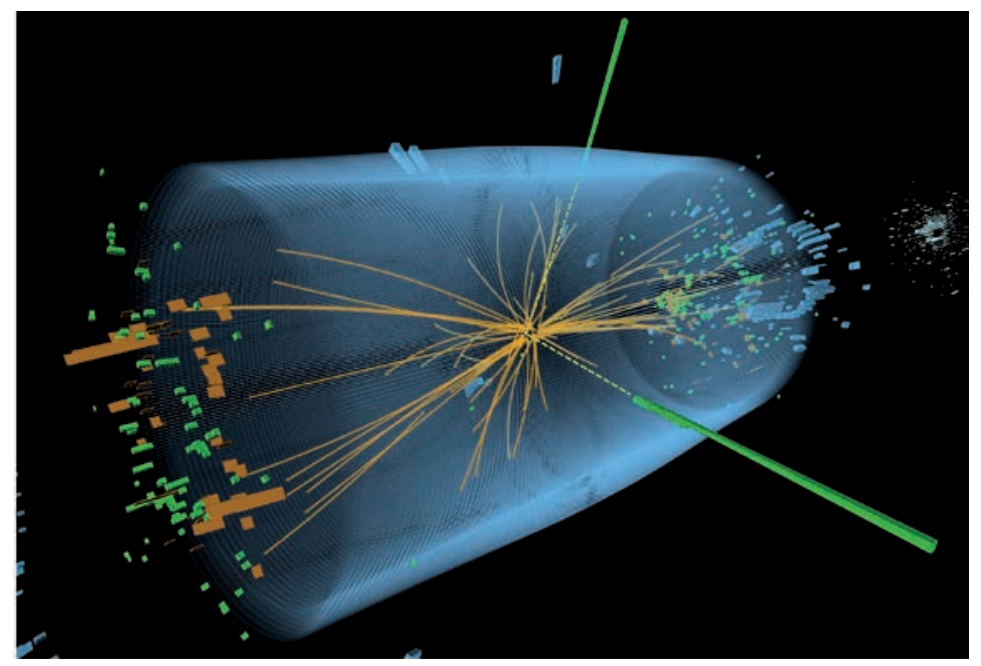

Fig. 3 - Un tipico evento a due fotoni come registrato dai rivelatori.

I fotoni sono le linee verdi. In figura sono visibili anche le trace lasciate da alter particelle prodotte insieme alla coppia di fotoni (fonte CERN).

\section{CONCLUSIONI}

L'attività di ricerca appena descritta è attività curiosity driven: i ricercatori che operano in questo campo sono spinti dal desiderio di capire la struttura della materia, dunque l'obiettivo principale è la conoscenza. D'altra parte è storicamente accertato che la ricerca di base produce spesso inattese applicazioni utili nella vita di tutti i giorni. Qui citiamo solo alcune di queste ricadute applicative, senza la pretesa di essere esaustivi. La Teoria della Relatività, speciale e generale, citata nel testo può apparire come una speculazione i cui effetti siano rivelabili solo in raffinati esperimenti dedicati. Ebbene non è così: un qualunque ricevitore GPS di uso quotidiano deve tener conto degli effetti relativistici, sia speciali che generali, sulla misura del tempo per poter operare; se non si tenesse conto di tali effetti, il GPS accumulerebbe un errore di circa dieci chilometri al giorno nella determinazione della posizione, cioè sarebbe 
completamente inutilizzabile. Tutti noi utilizziamo la posta elettronica e acquistiamo beni e servizi via il World Wide Web, che ha rappresentato una rivoluzione culturale, sociale ed economica. Esso è figlio del CERN, dove la tecnologia è stata sviluppata alla fine degli anni '80 del secolo scorso da Tim Berners-Lee allo scopo di consentire lo scambio di grandi moli di dati tra i laboratori di ricerca di tutto il mondo. Val la pena ricordare che la tecnologia World Wide Web è stata resa disponibile dal CERN al mondo intero, gratuitamente, il 30 aprile del 1993. Infine vanno ricordate le numerosissime applicazioni biomedicali sviluppate dalla ricerca nel campo della fisica delle interazioni fondamentali. Un esempio per tutte è lo CNAO (Centro Nazionale Adroterapia Oncologica), attivo nei pressi del Policlinico San Matteo di Pavia, dove vengono curati tumori profondi localizzati mediante l'uso di fasci di protoni e ioni pesanti sfruttando la tecnologia degli acceleratori di particelle.

\section{BIBLIOGRAFIA ESSENZIALE}

[1] http://www.nobelprize.org/nobel_prizes/physics/laureates/2013/

[2] P.W. Higgs, Broken symmetries, massless particles and gauge fields, in "Physics Letters", 12 (1964), p. 132; Broken symmetries, and the mass of the gauge bosons, in "Physical Review Letters", 13 (1964), p. 508.

[3] F. ENGLERT, R. BROUT, Broken symmetry and the mass of the gange vector mesons, in "Physical Review Letters", 13 (1964), p. 321.

[4] G.S. Guralnik, C.R. Hagen, T.W.B. KibBle, Global conservation laws and massless particles, Phys. Rev. Lett. 13 (1964) 585.

[5] A.A. MigDal, A.M. POLYAKOV, Spontaneous break-down of strong interaction symmetry and absence of massless particles, in "Soviet Physics JETP", 51 (1966), p. 135.

[6] Per un resoconto dettagliato dello sviluppo del Modello Standard delle interazioni fondamentali, articolato nella Teoria dell'Unificazione Elettrodebole e nella Cromodinamica Quantistica, nonché della sua formulazione attuale che include il calcolo degli effetti quantistici si veda ad esempio D. GRIFFITHS, Introduction to elementary particles, Wiley-VCH; 2nd edition (October 13, 2008), ISBN-10: 3527406018, ISBN-13: 978-3527406012.

[7] Si veda ad esempio G. Montagna, O. Nicrosini, G. Passarino, F. Piccinini, The Top quark and the Higgs boson mass from LEP SLC and CDF data, in "Physics Letters B", 335 (1994), pp. 484-489.

[8] ATLAS Collaboration, Observation of a new particle in the search for the Standard Model Higgs boson with the ATLAS detector at the LHC, in "Physics Letters B", 716 (2012), pp. 1-29.

[9] CMS Collaboration, Observation of a new boson at a mass of $125 \mathrm{GeV}$ with the CMS experiment at the LHC, in "Physics Letters B", 716 (2012), pp. 30-61. 